\title{
COMPARACIÓN DE CUATRO MÉTODOS FENOTÍPICOS PARA LA DETECCIÓN DE BETA-LACTAMASAS DE ESPECTRO EXTENDIDO
}

\author{
Lizet Lezameta ${ }^{1, a}$, Edgar Gonzáles-Escalante ${ }^{2, a}$, Jesús H. Tamariz',b,c,d
}

\begin{abstract}
RESUMEN
Objetivo. Comparar la eficacia de cuatro métodos fenotípicos para la identificación de cepas productoras de $\beta$-lactamasas de espectro extendido aisladas de urocultivos. Materiales y métodos. Estudio comparativo de corte transversal. Se analizó 147 cepas aisladas de urocultivos positivos para Escherichia coli, Klebsiella pneumoniae y Proteus mirabilis entre los meses de enero y febrero de 2009 en el Instituto Nacional de Salud del Niño, las cuales fueron sometidas a una prueba de tamizaje, en aquellas que resultaron positivas se realizó pruebas confirmatorias mediante los cuatro métodos fenotípicos evaluados. Resultados. De las 147 cepas, $43(29,3 \%)$ resultaron sospechosas en las pruebas de tamizaje. Con el método descrito por Clinical and Laboratory Standards Institute (CLSI) usado como "patrón de oro" para el estudio, resultaron 27 positivas $(62,8 \%)$, resultado similar a lo encontrado con el método de Jarlier. Por otro lado, con los métodos de Hodge y el tridimensional, $23(53,5 \%)$ resultaron positivas. La evaluación de los métodos confirmatorios frente al descrito por CLSI, mostró una sensibilidad y especificidad de $100 \%$ para el método de Jarlier; en el caso de los métodos de Hodge y método tridimensional se encontró una sensibilidad y especificidad de $85,2 \%$ y $100 \%$, respectivamente. Conclusiones. Los métodos evaluados mostraron en todos los casos una alta eficacia, sin presentar diferencias significativas, por lo que podrían utilizarse según las facilidades del laboratorio clínico involucrado; sin embargo dada las ventajas no técnicas, como costos, facilidad y factibilidad de su aplicación, se recomienda el empleo del método de Jarlier.
\end{abstract}

Palabras clave: Beta-Lactamasas; Farmacorresistencia microbiana; Técnicas y procedimientos diagnósticos; Cefalosporinas; Pediatría (fuente: DeCS BIREME).

\section{COMPARISON OF FOUR PHENOTYPIC METHODS TO DETECT EXTENDED-SPECTRUM BETALACTAMASES}

\begin{abstract}
Objective. To compare the efficacy of four phenotypic methods for the identification of strains producing extendedspectrum $\beta$-lactamases isolated from urine cultures. Materials and methods. Comparative cross-sectional study. 147 strains isolated from positive urine cultures for Escherichia coli, Klebsiella pneumoniae and Proteus mirabilis between January and February 2009 in the National Institute of Health of Children underwent a screening test, those which resulted positive were processed for confirmatory testing through the four phenotypic methods evaluated. Results. Out of the 147 strains, $43(29.3 \%)$ were suspicious in the screening tests. Using the method described by the Clinical and Laboratory Standards Institute (CLSI) as a reference standard for this study, 27 strains $(62.8 \%)$ were positive, with similar results using Jarlier's method. On the other hand, using Hodge's and tridimensional methods $23(53.5 \%)$ of samples were positive. The evaluation of the confirmation methods in comparison to the one described by CLSI, showed a sensitivity and specificity of $100 \%$ for Jarlier's method, on the other hand, for Hodge's and tridimensional methods we found a sensitivity of $85.2 \%$ and $100 \%$, respectively. Conclusions. All the evaluated methods showed a high efficacy, without significant differences, so they could all be used according to the available facilities of each clinical laboratory. Nevertheless, due to its advantages besides the technical aspect, like costs, easiness and feasibility of its application, we recommend the use of Jarlier's method.
\end{abstract}

Key words: Beta-Lactamases; Drug resistance, microbial; Diagnostic techniques and procedure; Cephalosponins; Pediatrics (source: MeSH NLM).

\section{INTRODUCCIÓN}

En la actualidad, los elevados niveles de resistencia a los antimicrobianos han incrementado los problemas relacionados con las enfermedades infecciosas, haciéndose cada vez más necesario el uso de nuevos antimicrobianos, más costosos y tóxicos para el paciente (1). Uno de los mecanismos de resistencias con mayor trascendencia clínica son las enzimas betalactamasas de espectro extendido (BLEE), codificadas por plásmidos,

Facultad de Medicina, Universidad Peruana Cayetano Heredia. Lima, Perú

Instituto Nacional de Salud del Niño. Lima, Perú.

a Tecnólogo Médico; ' Biólogo; c Magíster en Microbiología; ${ }^{d}$ Candidato a Doctor en Ciencias.

Recibido: 19-05-10 Aprobado: 01-09-10 
transposones e integrones (2). Por lo general, se presenta en bacilos Gram negativos como Escherichia coli, Klebsiella pneumoniae, Salmonella sp., Proteus sp., Citrobacter sp., Morganella morganni, Serratia marcescens, Shigella dysenteriae, Pseudomonas aeruginosa, Klebsiella oxytoca y Proteus mirabilis ${ }^{(3)}$.

Las BLEE tienen la capacidad de hidrolizar las oximinocefalosporinas como ceftriaxona (CRO), cefotaxima (CTX), ceftazidima (CAZ) y el aztreonam (ATM), quedando sensibles frente a las cefamicinas (cefoxitin, cefotetam) y carbapenems (imipenem, meropenem, ertapenem) ${ }^{(3,4)}$. Son clasificadas de acuerdo con dos sistemas generales: Ambler (1980) basada en la estructura molecular y la de Bush, Medeiros y Jacoby (1995) basada en similitudes funcionales (substratos) ${ }^{(4)}$. Se ha descrito diferentes familias de BLEE, como TEM y SHV y otros tipos como las cefotaximasas (CTX-M) descritas en 1989, las que se caracterizan por conferir resistencia de alto nivel a cefuroxima (CXM), cefotaxima (CTX) y cefepime (FEP) ${ }^{(4-6)}$. Las BLEE, constituyen un problema terapéutico y epidemiológico de gran magnitud, la presencia de estas cepas en las infecciones, conllevan a multirresistencia ya que son portadoras de otros genes que provocan resistencia cruzada a quinolonas, aminoglucósidos e incluso cotrimoxazol; de ahí la gran importancia de una adecuada y oportuna identificación ${ }^{(3,5)}$.

El Clinical and Laboratory Standards Institute (CLSI) recomienda como pruebas de tamizaje buscar la disminución de la inhibición en ATM, CTX, CAZ y CRO que permitan sospechar la presencia de BLEE en Escherichia coli y Klebsiella spp y como método confirmatorio se emplea cefotaxima y ceftazidima con y sin acido clavulánico, este último es un inhibidor de las BLEE ${ }^{(7-9)}$. Por su parte, el método de Jarlier ${ }^{(10)}$, basado en la sinergia entre los antibióticos betalactámicos (CAZ, CTX, CRO, ATM) colocados alrededor de un disco de amoxicilina/ácido clavulánico (20/10 $\mu \mathrm{g})$ (AMC), adicionalmente se ha determinado que el uso de cefalosporinas de cuarta generación como cefepime, facilita la detección de cepas BLEE con poca eficiencia hidrolítica ${ }^{(7,8,11,12)}$. Asimismo, el método de Hodge, usado para la determinación de mecanismos enzimáticos de resistencia, ha sido adaptado para identificar la presencia de $\beta$-lactamasas; dependiendo de los substratos que se utilicen, son útiles para cualquier tipo de ß-lactamasas (13). El método tridimensional descrito por Thomson et al., es un bioensayo, basado en la determinación de mecanismos enzimáticos de resistencia presente en microorganismos capaces de hidrolizar un antibiótico determinado ${ }^{(8,14,15)}$.

La diversidad en el grado de hidrólisis de los antibióticos por las $\beta$-lactamasas, conduce a la necesidad de evaluar la capacidad de los distintos métodos para la detección de cepas productoras de BLEE en nuestro medio, determinando de esta manera el método con mejores propiedades diagnósticas y económicas; lo que llevará a una oportuna identificación, con los consiguientes beneficios para el paciente y la comunidad y sobre esta base proponer las medidas de vigilancia para su detección e identificación ${ }^{(16,17)}$.

En el Perú no se ha reportado trabajos al respecto, además, no se cuenta con información de la real prevalencia de la resistencia antimicrobiana mediada por BLEE en bacterias Gram negativas, debido a la falta de estudios y la dificultad técnica para su detección. En ese sentido, este estudio tiene como objetivo comparar la eficacia de cuatro métodos fenotípicos para la identificación de cepas productoras de $\beta$-lactamasas de espectro extendido, aisladas de urocultivos en el Instituto Nacional de Salud del Niño (INSN), hospital pediátrico de alta complejidad y referencia nacional del Ministerio de Salud en Perú.

\section{MATERIALES Y MÉTODOS}

\section{DISEÑO DE ESTUDIO}

Se realizó un estudio comparativo de corte transversal sobre cepas aisladas de muestras de urocultivos en el Instituto Nacional de Salud del Niño (Lima, Perú) en los meses de enero y febrero de 2009.

\section{MATERIAL BIOLÓGICO}

Se incluyó 147 cultivos positivos de las especies Escherichia coli, Klebsiella pneumoniae y Proteus mirabilis recolectadas en el periodo de estudio. Realizada la identificación y verificada la pureza de las cepas, estas fueron conservadas a $-20{ }^{\circ} \mathrm{C}$ en caldo tripticasa soya con glicerol al $10 \%$, hasta su uso. Es preciso mencionar, que estas cepas fueron sometidas a una prueba de tamizaje basado en los criterios del CLSI ${ }^{(9,11)}$.

\section{PROCEDIMIENTOS}

Método de tamizaje para detección de $\beta$-lactamasas de espectro extendido según CLSI. Fue realizado por el método de disco difusión en agar Mueller Hinton (Britania) mediante la técnica de Baüer y Kirby, se usó discos de susceptibilidad antimicrobiana (Oxoid) de ATM $(30 \mu \mathrm{g}), \mathrm{CTX}(30 \mu \mathrm{g}), \mathrm{CAZ}(30 \mu \mathrm{g})$ y CRO $(30 \mu \mathrm{g})$; se utilizó como criterios de sospecha los diámetros: ATM $\leq$ $27 \mathrm{~mm}$; CTX $\leq 27 \mathrm{~mm}$; CAZ $\leq 22 \mathrm{~mm}$; y CRO $\leq 25 \mathrm{~mm}$. Se consideró sospechoso de BLEE, cuando la cepa presentó halos de inhibición iguales o inferiores a los 


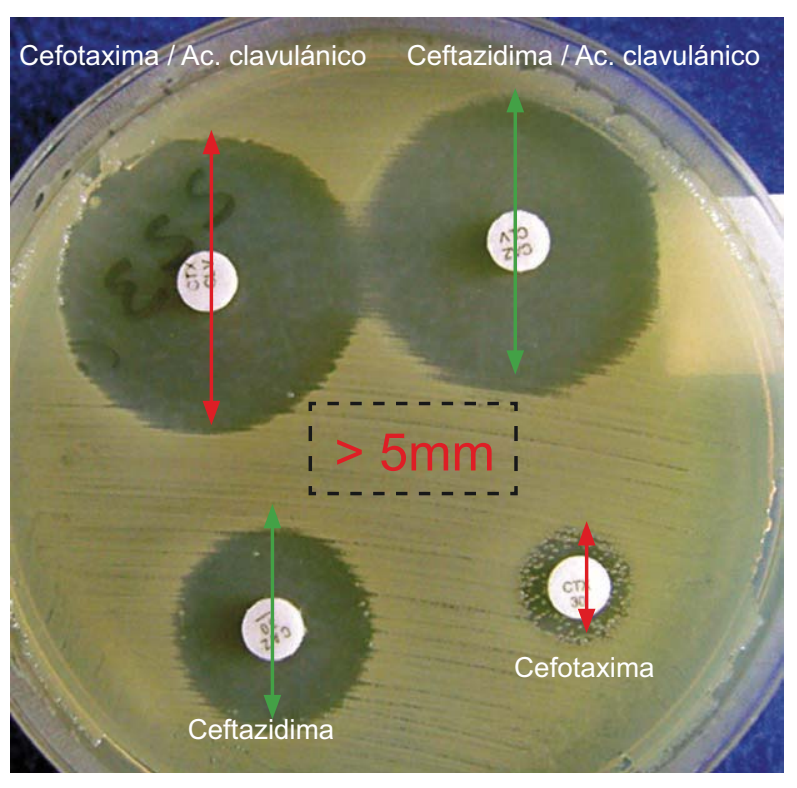

Figura 1. Prueba de disco combinado para la detección de BLEE. Se observa que la diferencia del halo de inhibición entre el disco de ceftazidima $(19 \mathrm{~mm})$ y el de ceftazidima/ácido clavulánico $(30 \mathrm{~mm})$ es de $11 \mathrm{~mm}$ y la diferencia entre los discos de cefotaxima ( $8 \mathrm{~mm}$ ) y cefotaxima/ acido clavulánico $(29 \mathrm{~mm})$ es de $21 \mathrm{~mm}$. Una diferencia mayor de $5 \mathrm{~mm}$ nos confirma la presencia de BLEE.

diámetros referidos, para al menos uno de los antibióticos $(8,9,18)$. Las cepas sospechosas fueron sometidas a las pruebas confirmatorias $(9,10,13,14)$.

Test confirmatorio BLEE - CLSI (método americano). Las placas de agar Mueller Hinton fueron inoculadas con las cepas sospechosas, para ello se siguió las recomendaciones del CLSI, colocándose discos de susceptibilidad antimicrobiana (Britania) de CAZ (30 $\mathrm{gg})$, ceftazidima/ácido clavulánico (CAZ/CAZ-CLA) $(30 / 10 \mu \mathrm{g})$, CTX (30 $\mu \mathrm{g})$, cefotaxima/ácido clavulánico (CTX/CXT-CLA) $(30 / 10 \mu \mathrm{g})$. Una diferencia mayor o igual a $5 \mathrm{~mm}$ en los halos de inhibición entre los discos de CAZ-CLA y CAZ solos o CXT-CLA y CTX, fue interpretada como resultado positivo ${ }^{(8,9,11)}$ (Figura 1).

Test confirmatorio BLEE - Método de Jarlier (Comité de la Sociedad Francesa de Microbiología). Las placas de agar Mueller Hinton fueron inoculadas con las cepas sospechosas, con una turbidez equivalente al tubo N. ${ }^{\circ} 0,5$ de la escala de Mc Farland. Se colocó un disco de amoxicilina/ácido clavulánico (AMC) $(20 / 10 \mu \mathrm{g})$ en el centro de una placa de Petri con agar Mueller Hinton y alrededor, a $25 \mathrm{~mm}$ de distancia, discos de CAZ (30 $\mu \mathrm{g} /$ $\mathrm{dL})$, CTX $(30 \mu \mathrm{g})$ y FEP $(30 \mu \mathrm{g})$. De manera opcional se analizó los discos de ATM $(30 \mu \mathrm{g})$ o CRO $(30 \mu \mathrm{g})$. La presencia de BLEE se manifestó por el efecto sinérgico del inhibidor y los discos -efecto de huevo, cola de pez o balón de futbol americano- ${ }^{(7,10,11)}$ (Figura 2).

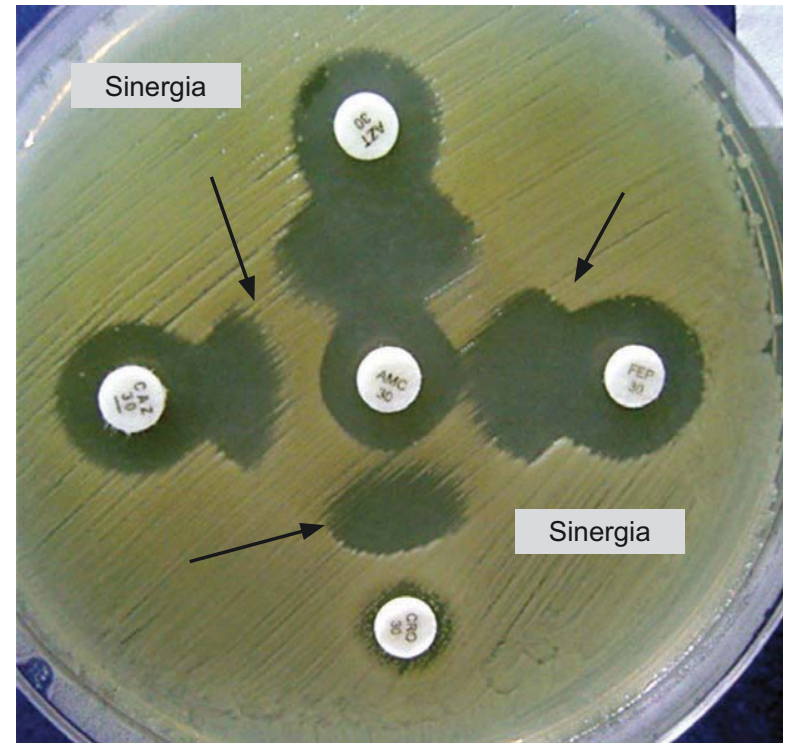

Figura 2. Método del doble disco para la detección de BLEE. Obsérvese el efecto sinérgico (tapón de corcho o distorsión de los halos de inhibición) (como indica la flecha) que se produce entre los discos de ceftriazona (CRO), cefepime (FEP), ceftazidima (CAZ) y aztreonam (AZM) con el disco central de amoxicilina/ácido clavulánico (AMC) Confirmación de la presencia de BLEE.

Método de Hodge para la determinación de BLEE. Se realizó una suspensión de la cepa de E. coli ATCC 25922, con turbidez equivalente al tubo $\mathrm{N} .^{\circ} 0,5$ de la escala de Mac Farland, esta suspensión se inoculó en una placa de agar Mueller Hinton. Se utilizó una placa por cada disco (CTX, CRO, ATM, FEP, CAZ) que fueron los sustratos a identificar. Se realizó una estría de $2 \mathrm{~cm}$ de la cepa a investigar, desde el centro hacia afuera del disco. La presencia de la enzima se identificó al observar una deformación del halo de inhibición de la cepa de $E$. coli ATCC 25922 al disco con el sustrato en forma de hendidura ${ }^{(13)}$ (Figura 3A).

Método tridimensional para la determinación de $B L E E$. Se realizó una suspensión de la cepa de $E$. coli ATCC 25922 con turbidez equivalente al tubo N. 0,5 de la escala de Mac Farland, esta suspensión se inoculó en una placa de agar Mueller Hinton, se colocó al centro de la placa Petri los discos conteniendo los sustratos por identificar (CTX, CRO, ATM, FEP, CAZ), se realizó un surco perpendicular al disco, en el extremo final se realizó un orificio de $2 \mathrm{~mm}$ en la cual se inoculó $20 \mu \mathrm{L}$ de la cepa problema de una suspensión equivalente al tubo 4 de la escala Mac Farland. La presencia de la enzima se identificó al observar una hendidura en el halo de inhibición, producto del crecimiento de la cepa indicadora de E. coli ATCC 25922 hacia el disco empleado como sustrato ${ }^{(14,15)}$ (Figura 3B). 

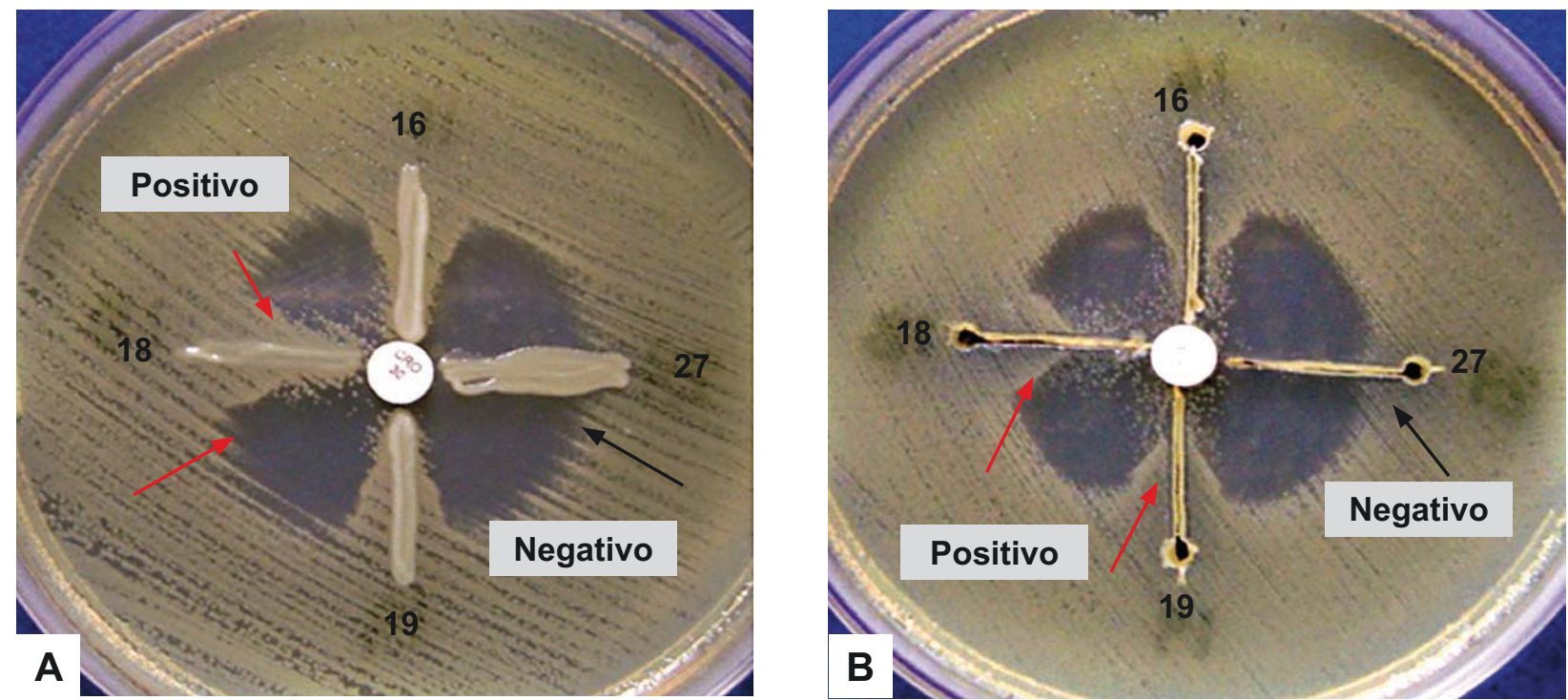

Figura 3.Se puede observar que la BLEE inactiva el disco de ceftriaxona (CRO) que difunde en el medio, por lo tanto no hay suficiente CRO para inhibir la cepa de referencia (E.coli ATCC 25922) y se produce la zona de inhibición (cepa 16, 18,19 BLEE positivas, cepa 27 BLEE negativa), según método de Hodge (A) y tridimensional (B).

\section{CONTROL DE CALIDAD}

Se probó como control negativo, cepas de E. coli ATCC 25922 BLEE (-) y como control positivo K. pneumoniae ATCC 700603 BLEE (+), el control de calidad de los discos empleados tanto para el tamizaje como para los cuatro métodos fue realizado usando cepas ATCC de E. coli 25922, E. coli 35218 y Pseudomonas aeruginosa 28573, cumpliendo satisfactoriamente con los criterios establecidos por el CLSI ${ }^{(9)}$.

\section{ANÁLISIS ESTADÍSTICO}

Los datos fueron procesados en el programa Microsoft Excel $2007 \AA$, se calculó la sensibilidad y especificidad con los intervalos de confianza al $95 \%$ usando el programa estadístico Epidat v.3.1.

\section{RESULTADOS}

Se analizó un total de 147 cepas bacterianas, 130 cepas de E. coli $(88,4 \%), 11$ de $K$. pneumoniae $(7,5 \%)$ y 6 de
Proteus mirabilis $(7,1 \%)$. De las 147 cepas estudiadas, $43(29,3 \%)$ resultaron sospechosas para la producción de BLEE en la prueba de tamizaje.

Al evaluar estos resultados a través del método americano -patrón de oro en el estudio- de las 43 cepas sospechosas según el tamizaje, $27(62,8 \%)$ fueron confirmadas para la producción de BLEE. Con el método de Jarlier se observó resultados similares; 27 cepas $(62,8 \%)$ resultaron positivas para BLEE, con una sensibilidad y especificidad del $100 \%$. Sin embargo, los resultados obtenidos con los métodos de Hodge y tridimensional, de las 43 cepas estudiadas, $23(53,5 \%)$ resultaron positivas para BLEE por ambos métodos, con lo que se calculó una sensibilidad de $85,1 \%$ (IC95\%: 69,9 a 100) y una especificidad del $100 \%$ (IC95\%: 96,9 a 100) (Tabla 1).

Los resultados indican que CTX detectó la presencia de BLEE más eficazmente que los otros antimicrobianos, se observó mayor sensibilidad en el método tridimensional con respecto al método de Hodge; pero con igual especificidad (Tabla 2).

Tabla 1. Evaluación de las propiedades diagnósticas de los métodos confirmatorios para la detección de BLEE, con referencia al método americano como patrón de oro $(n=27)$.

\begin{tabular}{lcccccccc}
\hline Métodos & VP & FN & S (IC95\%) & PFN & VN & FP & E (IC95\%) & PFP \\
\hline Jarlier & 27 & 0 & $100(98,2-100)$ & 0 & 16 & 0 & $100(96,9-100)$ & 0 \\
Hodge & 23 & 4 & $85,2(69,9-100)$ & 14,8 & 16 & 0 & $100(96,9-100)$ & 0 \\
Tridimensional & 23 & 4 & $85,2(69,9-100)$ & 14,8 & 16 & 0 & $100(96,9-100)$ & 0 \\
\hline
\end{tabular}

VP: verdaderos positivos; FN: falsos negativos; S: sensibilidad; PFN: proporción de falsos negativos; VN: verdaderos negativos; FP: falsos positivos; E: especificidad; PFP: proporción de falsos positivos. 
Tabla 2. Evaluación de las propiedades diagnósticas de cada antimicrobiano empleado en el método de Hodge y método tridimensional para la detección de $\beta$-lactamasas de espectro extendido con referencia al método americano.

\begin{tabular}{|c|c|c|c|c|c|c|c|c|}
\hline \multirow{2}{*}{ Disco de Antibiograma } & \multicolumn{4}{|c|}{ Método de Hodge } & \multicolumn{4}{|c|}{ Método Tridimensional } \\
\hline & VP & FN & $\mathbf{S}$ & PFN & VP & FN & $\mathbf{S}$ & PFN \\
\hline Aztreonam (ATM) & 3 & 24 & 11,1 & 88,9 & 4 & 23 & 14,8 & 85,2 \\
\hline Ceftazidima (CAZ) & 0 & 27 & 0 & 100 & 1 & 26 & 3,7 & 96,3 \\
\hline Cefotaxima (CTX) & 22 & 5 & 81,5 & 18,5 & 23 & 4 & 85,2 & 14,8 \\
\hline Ceftriaxona (CRO) & 20 & 7 & 74,1 & 25,9 & 20 & 7 & 74,1 & 25,9 \\
\hline Cefepime (FEP) & 12 & 15 & 44,4 & 55,6 & 15 & 12 & 55,6 & 44,5 \\
\hline
\end{tabular}

Especificidad: $100 \%$; verdaderos negativos: 16; falsos positivos: 0; proporción de falsos positivos: $0 \%$.

VP: verdaderos positivos; FN: falsos negativos; S: sensibilidad; PFN: proporción de falsos negativos.

Al evaluar cada antimicrobiano empleado en el método de tamizaje, se observó mayor capacidad de detección con los antimicrobianos CRO y CTX, con una sensibilidad de $100 \%$ para ambos antimicrobianos (Tabla 3 ).

\section{DISCUSIÓN}

La identificación de BLEE debe ser una prioridad en los laboratorios de microbiología clínica a fin de posibilitar un abordaje terapéutico adecuado, para lo cual existe una diversidad de opciones: métodos fenotípicos, genotípicos, presuntivos y confirmatorios, todos ellos con ventajas y desventajas ${ }^{(16,18,19)}$. Las pruebas fenotípicas constituyen una estrategia muy práctica, económica y accesible, en tal sentido, su implementación debería ser ampliamente difundida en los laboratorios microbiológicos de nuestro medio.

En otros países se ha realizado algunos estudios similares al nuestro, como el realizado en India, donde se comparó el método de sinergia de doble disco y el método tridimensional; la conclusión fue que el último de ellos dio $93 \%$ de sensibilidad frente al $85 \%$ del primero ${ }^{(15)}$. En Bélgica, se comparó en setenta aislamientos clínicos de Escherichia coli y Klebsiella spp, tres métodos de tamizaje para la detección y prevalencia de BLEE por el método de doble disco, método tridimensional y E-test-BLEE, en este caso se concluyó que la sensibilidad de las pruebas fue de $96,9 \%, 90,6 \%$ y $81,2 \%$, respectivamente ${ }^{(20)}$.

En el presente estudio, el método americano fue considerado como "patrón de oro" para la detección de BLEE, siguiendo las recomendaciones del CLSI, entidad que recomienda su empleo como método de referencia en base a su buena sensibilidad y especificidad, reafirmado por diferentes estudios $(7,8,9,21)$.

El método de Jarlier demostró ser tan sensible y específico como el patrón de oro, presenta, además, ventajas competitivas que lo convierten en una buena opción como prueba confirmatoria, ello debido a que emplea los mismos materiales y procedimientos que un antibiograma de rutina, y a diferencia del método americano, no emplea discos de susceptibilidad adicionales; asimismo, no requiere pruebas de tamizaje, lo que disminuye el tiempo de respuesta, implicando menores costos de procesamiento; se suma a ello, el ser operativamente más sencillo, sobre todo cuando se requiere evaluar un número elevado de muestras. La desventaja del método de Jarlier es que modificaciones en la ubicación y distancia de los discos, puede conducir a resultados erróneos en personal no experimentado.

El método de Hodge y el tridimensional demostraron una buena sensibilidad y especificidad, puesto que son

Tabla 3. Evaluación de la sensibilidad (S) y especificidad (E) de cada antimicrobiano empleado en el método de tamizaje para la detección de $\beta$-lactamasas de espectro extendido con referencia al método americano $(n=43)$.

\begin{tabular}{lccccccrcrr}
\hline Disco de antibiograma & TP & TN & VP & FN & S (\%) & PFN & VN & FP & E (\%) & PFP \\
\hline Aztreonam (ATM) & 33 & 10 & 26 & 1 & $(96,3)$ & 3,7 & 9 & 7 & $(56,3)$ & 43,8 \\
Ceftazidima (CAZ) & 21 & 22 & 20 & 7 & $(74,1)$ & 25,9 & 15 & 1 & $(93,8)$ & 6,3 \\
Cefotaxima (CTX) & 34 & 9 & 27 & 0 & $(100)$ & 0,0 & 9 & 7 & $(56,3)$ & 43,8 \\
Ceftriaxona (CRO) & 35 & 8 & 27 & 0 & $(100)$ & 0,0 & 8 & 8 & $(50,0)$ & 50,0 \\
\hline
\end{tabular}

TP: total de positivos; TN: total de negativos; VP: verdaderos positivos; FN: falsos negativos; PFN: proporción de falsos negativos; VN: verdaderos negativos; FP: falsos positivos; PFP: proporción de falsos positivos. 
técnicas que pueden usar diferentes antibióticos, lo que permitiría la identificación del mecanismo enzimático y el posible gen involucrado; se constituye así en una herramienta útil, sobre todo en laboratorios de referencia. No obstante, tienen la desventaja de requerir para su ejecución la cepa de referencia E. coli ATCC 25922, con el consecuente aumento de los costos operativos, además, se requiere adiestramiento especial para la ejecución e interpretación de los resultados, ya que en muchos casos la cepa en estudio puede ser productora de BLEE, pero ser expresada en bajas concentraciones, debido a que la expresión fenotípica de enzimas está sujeta a una serie de factores reguladores, que podrían conducir a resultados no evidentes.

Al analizar la sensibilidad y especificidad de los discos empleados tanto en el método de tamizaje, el método de Hodge y el método tridimensional, se aprecia que el disco de CTX fue muy sensible respecto al disco de $\mathrm{CAZ}$, detectando la presencia de BLEE en las cepas estudiadas; además, fue equivalente a la combinación de discos de CTX/CTX-CLA propuesto por el CLSI. Esto no debe interpretarse de manera definitiva como una ventaja de los antibióticos mencionados, sino que responde a los mecanismos de resistencia propia de las cepas de nuestro estudio, lo cual puede cambiar a lo largo del tiempo o en otros ambientes hospitalarios, por lo que es recomendable el desarrollo de estudios en otro marco temporal y geográfico. Bajo lo encontrado en este estudio, puede deducirse que fenotípicamente las cepas estudiadas se inclinan al perfil de ser productoras de la enzima cefotaximasas (gen tipo CTX-M) con una mayor actividad sobre CTX en la cual se observó una sensibilidad del $100 \%$, sin embargo al utilizar el disco de CAZ se observó un importante descenso de la sensibilidad; la confirmación de estos hallazgos requiere la aplicación de pruebas moleculares. Se debe considerar la presencia de otros mecanismos de resistencia, como otras enzimas $\beta$-lactamasas, diferentes a las BLEE, que pueden dificultar la interpretación de los resultados, ello ratifica la importancia de la adecuada elección del método confirmatorio.

Respecto a los métodos de Hodge y tridimensional es importante mencionar su limitación en la detección de BLEE en $P$. mirabilis, debido al fenómeno de enjambre -del inglés swarming- propio de esta bacteria, lo que conduce a errores de interpretación.

Finalmente, el método americano es un método sencillo y práctico. Sin embargo, para bacterias que además de producir BLEE produzcan otros mecanismos de resistencia como la producción de de $\beta$-lactamasas tipo AmpC, dificulta la identificación de la presencia de BLEE, que si puede ser detectada con otros métodos.
Es importante reconocer que la investigación presenta limitaciones al no haberse calculado el tamaño muestral previo requerido para efectuar un estudio de prueba diagnóstica, asimismo, existe un sesgo de selección dado que se tratan de muestras hospitalarias, aunque las bacterias de este tipo son predominantemente de origen nosocomial.

En conclusión, los métodos evaluados mostraron una elevada eficacia, sin presentar diferencias significativas, por lo que podrían usarse según las facilidades del laboratorio clínico; sin embargo, debido a las ventajas no técnicas, como costos y factibilidad de su aplicación; como producto del presente estudio se recomienda el empleo del método de Jarlier.

\section{AGRADECIMIENTOS}

A la Dra. Lilian Patiño Gabriel, Jefa del Servicio de Microbiología del Instituto Nacional de Salud del Niño (INSN) que brindó las facilidades del caso para la realización práctica del presente trabajo. Al Dr. Rito Zerpa por la revisión final del manuscrito. A los licenciados en tecnología médica José María Olivo, Cristhian Villar y al personal del Servicio de Microbiología del INSN. Al ingeniero Wilfredo Mormontoy por la asesoría estadística.

\section{Fuentes de Financiamiento}

Autofinanciado.

\section{Conflictos de Interés}

Los autores declaran no tener conflictos de interés en la publicación de este artículo.

\section{REFERENCIAS BIBLIOGRÁFICAS}

1. Labarca L Jaime, Araos B Rafael. Resistencia antimicrobiana: Problema en aumento y soluciones escasas. Rev Chil Infect. 2009;26(Supl 1):8-9.

2. Cano ME, Domínguez MA, Ezpeleta C, Padilla B, Arellano ER, Martínez-Martínez L. Cultivos de vigilancia epidemiológica de bacterias resistentes a los antimicrobianos de interés nosocomial. Enf Infecc Microbiol Clin. 2008;26(4):220-9.

3. Ramos-Godínez A, Hernández-Pedroso W, NodarseHernández R, Padrón-Sánchez A, De Armas-Alonso E, Del Rosario-Cruz L. Detección precoz de enterobacterias productoras de betalactamasas de espectro extendido en pacientes graves. Rev Cub Med Int Emerg. 2006;5(1):294301.

4. Paterson DL, Bonomo RA. Extended-spectrum betalactamases: a clinical update. Clin Microbiol Rev. 2005;18(4):657-86.

5. Cavalieri SJ, Harbeck RJ, McCarter YS, Ortez JH, Rankin ID, L. Sautter RL, et al. Manual de pruebas de 
susceptibilidad antimicrobiana. Seattle: University of Washington; 2005.

6. Garza-Ramos U, Silva-Sánchez J, Martínez-Romero E. Genética y genómica enfocadas en el estudio de la resistencia bacteriana. Salud Publica Mex 2009;51(Supl 3):S439-46.

7. Famiglietti A, Quinteros $M$, Vázquez M, Marín M, Nicola F, Radice M y col. Consenso sobre las pruebas de sensibilidad a los antimicrobianos en Enterobacteriaceae. Rev Arg Microb. 2005;37(1):57-66.

8. Oliver A, Cantón R. Enterobacterias productoras de ß-Lactamasas plasmídicas de espectro extendido. Madrid: Sociedad Española de Enfermedades Infecciosas y Microbiología Clínica (SEIMC); 2003.

9. Clinical and Laboratory Standard Institute. Performance standards for antimicrobial susceptibility testing. Nineteenth informational supplement M100-S19. Wayne, Pennsylvania: Clinical and Laboratory Standard Institute; 2009.

10. Jarlier V, Nicolas MH, Fournier G, Philippon A. Extended broad-spectrum beta-lactamases conferring transferable resistance to newer beta-lactam agents in Enterobacteriaceae: hospital prevalence and susceptibility patterns. Rev Infect Dis. 1988;10(4):867-78.

11. Calderón ER, Yagui MM, Sacsaquispe CR. Manual de procedimientos para la prueba de sensibilidad antimicrobiana por el método de difusión disco. Lima: Instituto Nacional de Salud; 2002.

12. Drieux L, Brossier F, Sougakoff W, Jarlier V. Phenotypic detection of extended-spectrum beta-lactamase production in Enterobacteriaceae: review and bench guide. Clin Microbiol Infect. 2008;14 (Suppl 1):90-103.

13. Hodge W, Ciak J, Tramont EC. Simple method for detection of penicillinase-producing Neisseria gonorrhoeae. J Clin Microbiol. 1978;7(1):102-3.

14. Thomson KS, Sanders CC. Detection of extendedspectrum beta-lactamases in members of the family Enterobacteriaceae: comparison of the double-disk and three-dimensional tests. Antimicrob Agents Chemother. 1992;36(9):1877-82.
15. Menon T, Bindu D, Kumar CP, Nalini S, Thirunarayan MA. Comparison of double disc and three dimensional methods to screen for ESBL producers in a tertiary care hospital. Indian J Med Microbiol. 2006;24(2):117-20.

16. Sánchez B. Betalactamasas de espectro extendido. Rev Electr de Med Intensiva [Revista en Internet]. 2004 [Fecha de acceso: 11 de agosto de 2004];4(8):[13 pantallas]. Disponible en: http://remi.uninet.edu/2004/08/REMIC06. $\mathrm{htm}$

17. Perozo AJ, Castellano MJ. Detección de betalactamasas de espectro extendido en cepas de la familia Enterobacteriaceae. Kasmera. 2009;37(1):25-37.

18. Bauer AW, Kirby WM, Sherris JC, Turck M. Antibiotic susceptibility testing by a standardized single disk method. Am J Clin Pathol 1966; 45(4):493-6.

19. Bertona E, Radice M, Rodríguez C, Barberis C, Vay C, Famiglietti A, et al. Caracterización fenotípica y genotípica de la resistencia enzimática a las cefalosporinas de tercera generación en Enterobacter spp. Rev argent microbiol. 2005;37(4):203-8.

20. Vercauteren E, Descheemaeker P, Leven M, Sanders CC, Goossens H. Comparison of screening methods for detection of extended-spectrum beta-lactamases and their prevalence among blood isolates of Escherichia coli and Klebsiella spp. in a Belgian teaching hospital. J Clin Microbiol. 1997;35(9):2191-7.

21. Camacho ML, Perozo MA, Castellano GM, Bermúdez NE, Harris SB. Métodos fenotípicos para la detección de betalactamasas de espectro extendido en cepas de Escherichia coli y Klebsiella pneumoniae. Rev Soc Ven Microbiol 2004;24(1-2):132-9.

Correspondencia: Jesus H. Tamariz Ortiz

Dirección: Av. Honorio Delgado N. ${ }^{\circ} 430$, San Martín de Porras, Lima 31, Lima, Perú.

Teléfono: 511-3190000 anexo: 2345

Correo electrónico: jesus.tamariz@upch.pe

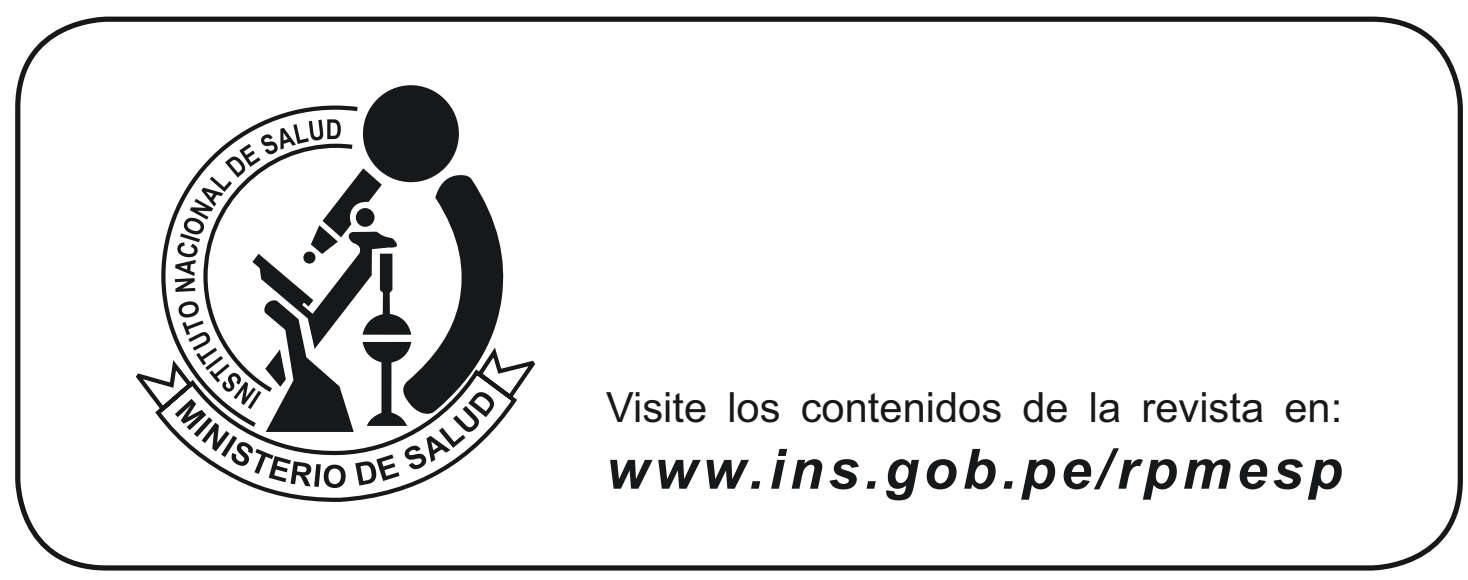

